\title{
Estimation of the drift eliminator efficiency using numerical and experimental methods
}

\author{
Jiři Stodůlka ${ }^{1, a}$, Rut Vitkovičová ${ }^{1}$ \\ ${ }^{1}$ CTU in Prague, FME, Department of Fluid Dynamics and Thermodynamics, Prague, Czech Republic
}

\begin{abstract}
The purpose of the drift eliminators is to prevent water from escaping in significant amounts the cooling tower. They are designed to catch the droplets dragged by the tower draft and the efficiency given by the shape of the eliminator is the main evaluation criteria. The ability to eliminate the escaping water droplets is studied using CFD and using the experimental IPI method.
\end{abstract}

\section{Introduction}

This paper deals with the simulation of the flow in the optimized multifunctional cooling tower drift eliminator in order to obtain it's functional parameters. The flow field which is directly connected with the efficiency estimation are both simulated using CFD code ANSYS Fluent which was previously validated by the PIV visualization method and by experimental pressure loss evaluation on the prototype model. A further analysis in terms of the efficiency itself has been done based on the validated flow field using again the CFD methods and then compared with experimental IPI method.

\section{Drift eliminators}

Beside the water distribution system and cooling panels are the eliminators another important functional part of the cooling tower [1], whose arrangement is schematically described in the figure 1 .

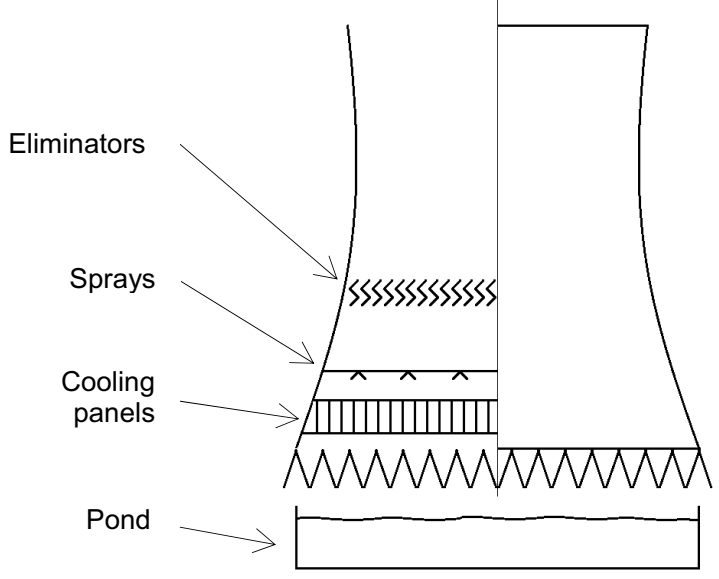

Figure 1. Natural draft cooling tower scheme.
The main purpose of these curved shape panels is to rapidly reduce the total mass flow carried away by air exiting the tower (drift) using their shape to change the flow direction causing enough inertia forces, so that the fluid droplets can't follow the air stream and hit the wall tickeling down as a film and drop back to the tower. The amount of water contained in both the form of vapor generated during the cooling process [2] and liquid droplets generated by condensation, but also very small diameter droplets drifted away from the spraying zone [3] escaping the tower, was estimated at approx. 0.001 $\mathrm{m}^{3} / \mathrm{hr} / \mathrm{MW}$ back in the year 2000 . This number may seem small, but taking into account high power outputs of modern energetic open recirculation systems, the total mass flow escaping is significant and that plays an important role in economical and also ecological function of the system. To restrict all the unpleasant side effects, the eliminator manufacturers have to guarantee nowadays to reduce the drift according to standards to maximum of $0.0005 \%$

The most commonly used types are the grid eliminators, made of thin profiled slats connected together. Example of such eliminator is shown in the figure 2 .
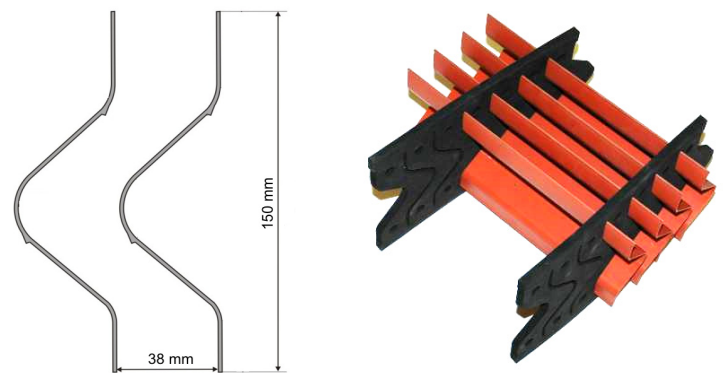

Figure 2. Grid type eliminator.

\footnotetext{
$\overline{{ }^{a} \text { Corresponding author: jiri.stodulka }} @$ fs.cvut.cz
} 
Another relatively new type is the eliminator with moisture recovery system. This type combines two principles; the first is the elimination of liquid phase similarly like the grid type eliminator and simultaneously a function of heat exchanger. These eliminators are bigger and hollow, to allow outer air with lower temperature pass through to cool down the escaping moist air ending in condensation of the moist air. A lot of attention is paid nowadays to improve the behaviour of these multifunctional eliminators in order to save more and more amount of water escaping from the evaporative cooling systems.

\section{Design}

Shape of every eliminator has to fulfil three main requirements. These are maximal efficiency in terms of caught water droplets, minimal pressure loss and minimal amount of used material. Unfortunately all three requirements are against each other, so it is necessary to find appropriate balance between them. The shape of investigated eliminator row is in the figure 3 .

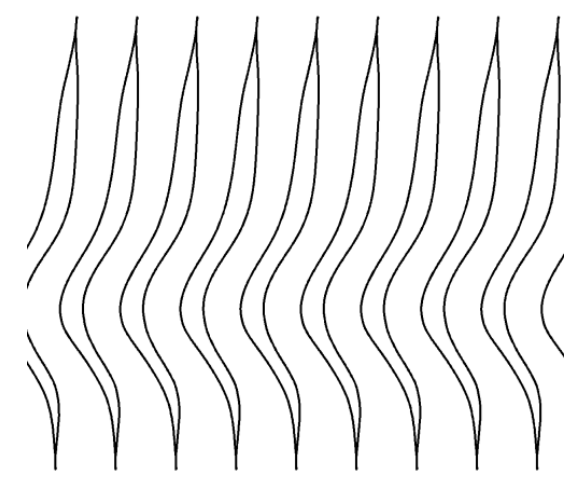

Figure 3. Eliminator shape.

The overall height of the eliminator is $450 \mathrm{~mm}$, while distance between neighbouring ones is $60 \mathrm{~mm}$.

\section{Flow simulation and validation}

For the numerical simulations the commercial CFD code ANSYS Fluent was used. Schematic view on the model and boundary conditions setup is in the figure 4 .

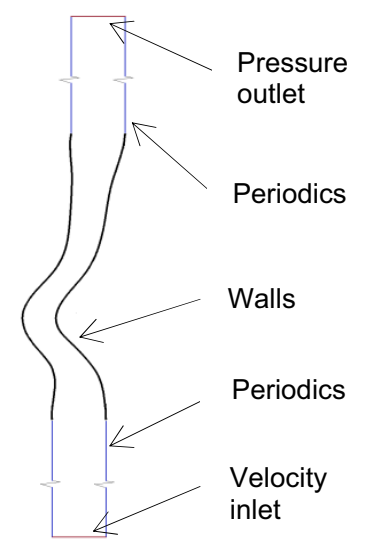

Figure 4. Computational model description.
Velocity inlet values vary depending on concrete cooling tower conditions from 1.5 to $4 \mathrm{~m} / \mathrm{s}$ with the turbulent intensity of $2 \%$. Pressure at the outlet is set to be atmospheric. Due to relatively simple geometry the mapped $2 \mathrm{~d}$ quad mesh was used for the whole domain with total count of approx. 100000 elements. Implemented sst $\mathrm{k}-\omega$ turbulence model was used, what leads to demand for fine mesh close to the walls. The values of $\mathrm{Y}+$ oscillate around 0.5 for all velocity modes. The detail of the mesh is shown in figure 5 .

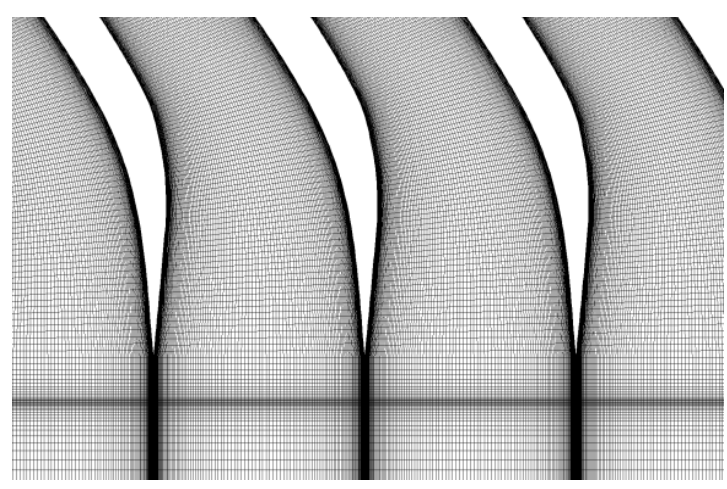

Figure 5. Quad computational mesh.

Results in form of velocity contours for inlet mode $2.6 \mathrm{~m} \mathrm{~s}^{-1}$ are shown in the figure 6. Except the trailing edge of the eliminator, no other separation occurs resulting in reasonable pressure loss. The flow is also significantly accelerated, so that droplets elimination can be expected in curved areas.

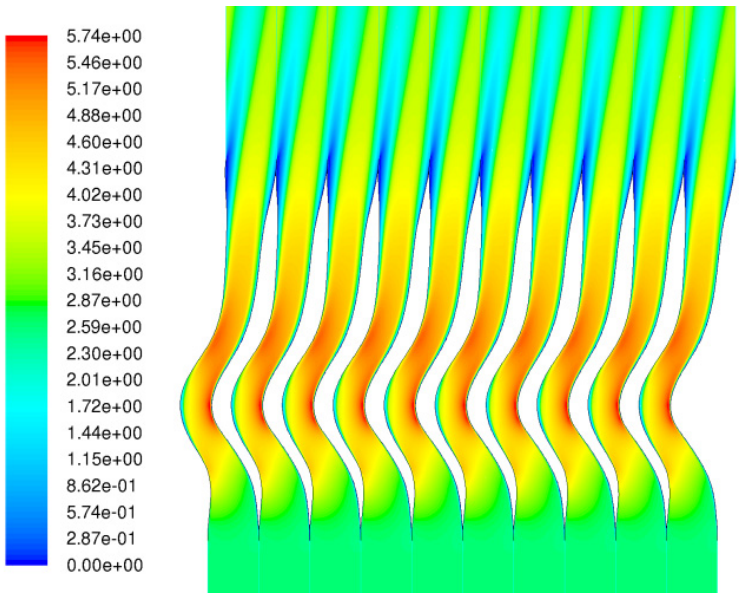

Figure 6. Contours of velocity magnitude $\left(\mathrm{m} \mathrm{s}^{-1}\right)$

For experimental evaluation of the flow field the PIV method was used. Models of eliminator were placed in wind tunnel prepared for optical visualisation methods; see figure 7. The inlet velocity was approximately $3.2 \mathrm{~m}$ $\mathrm{s}^{-1}$ and the CFD boundary condition for comparison simulation was adjusted according to that. Pressure loss was simply evaluated by pressure difference in front of and behind the eliminator in bigger classical open aerodynamic tunnel to minimize the effect of tunnel itself. 


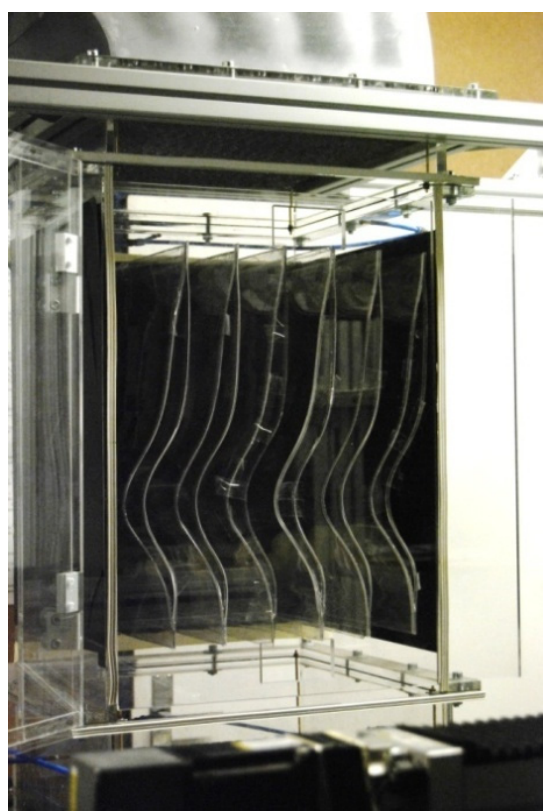

Figure 7. Experimental model.

For a direct comparison between experimental and numerical results, the data from both were analyzed using Matlab. Data in form of velocity component contours were plotted on the same grid and then subtracted from each other to see the differences. The evaluation of the difference between CFD and PIV for the x(right) and $\mathrm{y}(\mathrm{left})$-velocity components is shown in the figure 8 .

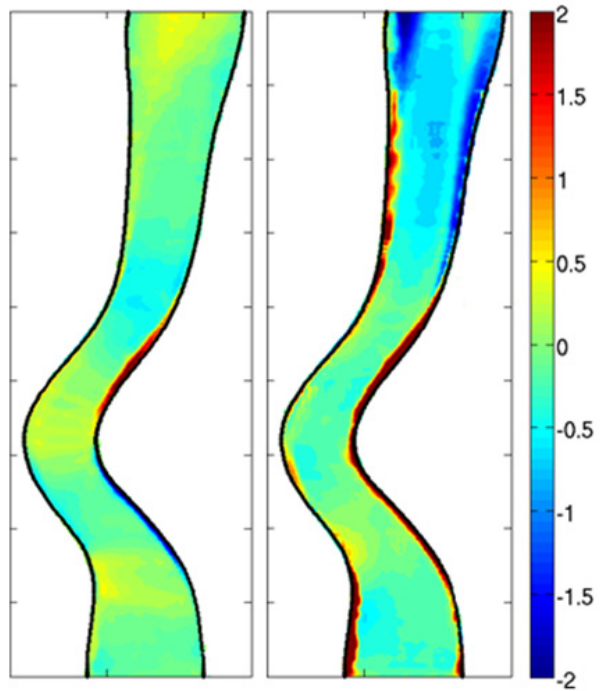

Figure 8. Velocity components comparison

From this figure is obvious a good accordance between these two approaches, at least in the main centre flow, where the difference ranges only in few tenths of $\mathrm{m} / \mathrm{s}$, what was also confirmed from velocity profiles. Near walls is the difference more dramatic, but that can be due to limits of the PIV near strongly reflective boundary and direct real and model geometry comparison. Major inconsistency appears only at the upper part of the eliminator, where mainly y-components exhibit significant disparity. That was probably caused by inconsistence between numerical and physical eliminator model and the finite dimension of the wind tunnel can play its role as well. However, this area is no more important in terms of the main function of the eliminator, while all possible droplet separation happens in the curved section.Considering the geometry differeces, the CFD is slightly underpredicting in direct comparison, but the numerical results are still within the errorbars. In terms of pressure loss, the maximal value for highest velocity mode $\left(4 \mathrm{~m} \mathrm{~s}^{-1}\right)$ is $5.9 \mathrm{~Pa}$, for the lowest mode $\left(1.5 \mathrm{~m} \mathrm{~s}^{-1}\right)$ is $1.2 \mathrm{~Pa}$. The experimental pressure loss estimation confirmed these values as well as all the numbers from the numerical simulation were inside the error tolerance of the experimental setup. The further details can be also found in [4].

\section{Efficiency estimation}

According to previous results it can be said, that in terms of the flow filed the numerical model predicts the behavior sufficiently. The efficiency then can be calculated using the same code with built-in Discrete phase modeler (DPM). This model computes the trajectory of solid or liquid particles in main fluid flow according to the forces equilibrium:

$$
\begin{gathered}
m_{p} \frac{d u_{p}}{d t}=F_{\text {drag }}+F_{\text {pressure }}+ \\
F_{\text {virtual mass }}+F_{\text {gravity }}+F_{\text {other }} .
\end{gathered}
$$

Where the particular forces are the drag forces

$$
F_{\text {drag }}=\frac{\rho A_{p}}{2} C_{d}\left\|u-u_{p}\right\|\left(u-u_{p}\right),
$$

the pressure forces

$$
F_{\text {pressure }}=\int-p d A,
$$

the forces of virtual mass

$$
F_{\text {virtual mass }}=\frac{m_{p}}{2} \frac{\rho}{\rho_{p}} \frac{d\left(u-u_{p}\right)}{d t}
$$

and the gravity forces.

$$
F_{\text {gravity }}=m_{p} \frac{\rho-\rho_{p}}{\rho_{p}} g .
$$

The individual particles cannot interact between each other but it is possible to simulate the interaction between particles and the main flow, but in this case, this option was not used. The particles with properties of water were released from the inlet boundary condition and their behavior on the walls was set to "trap" option, that means that when the particle touches the wall, it simply disappears. On the outlet, the particles are allowed to escape from the domain. The overall efficiency is then the ratio of number of trapped to number of released. Calculated trajectories for different particle diameter for inlet velocity $2.6 \mathrm{~m} / \mathrm{s}$ are shown in the figure 9 .

The particular efficiencies are marked in the efficiency-diameter graph for all investigated velocities. In order to obtain continual distribution, the points in the graph were fitted with the funcion

$$
\eta=1-\exp \left(a D^{b}\right)
$$




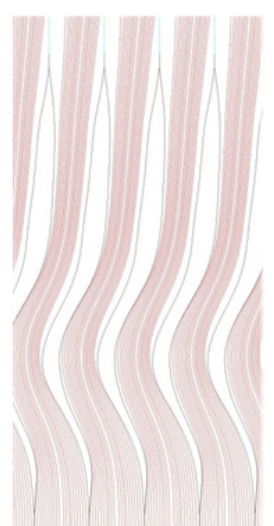

$20 \mu \mathrm{m}$

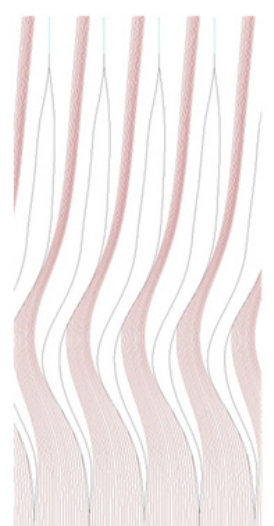

$50 \mu \mathrm{m}$

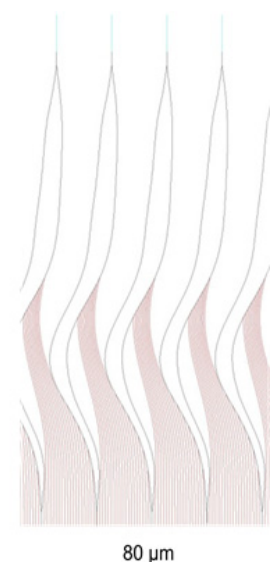

$80 \mu \mathrm{m}$
Figure 9. Particle trajectories.

The final efficiency diagram for different velocities is shown in the figure 10 .

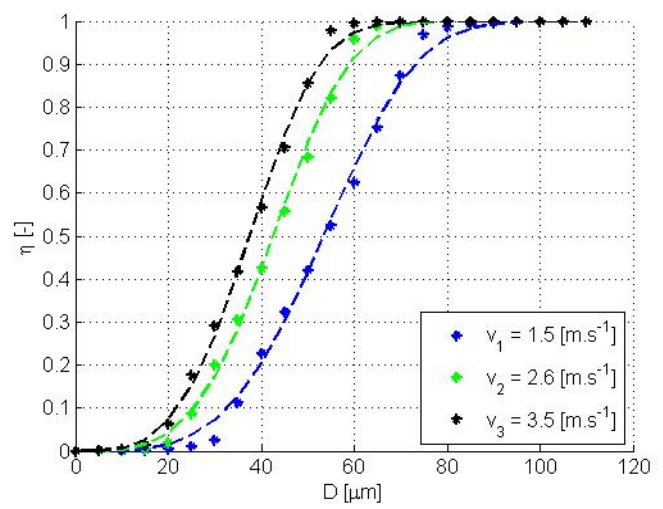

Figure 10. Eliminator efficiency.

This graph shows that for any inlet velocity, almost all particles smaller than 10 microns in diameter are dragged away. After this point, the eliminators starts to eliminate accordingly to the velocity magnitude - for higher velocities is the efficiency higher. For the slowest regime the biggest particles should not exceed 100 microns. Considering the fact that the absolute majority of the particles in the cooling tower are in range of tenths of millimeters or millimeters, is the efficiency for this special low drag eliminator still very good.

These numbers were also verified using experimental methods, concretely using the Interferometric particle imaging or IPI. This optical method is based on capturing the interfering beams reflected from transparent particle. More about this method and the particular setup can be found in [5]. The beam of coherent light incidents on the particle and is partly reflected and partly passes through and refracts. These two beams then interfere in the illuminated area and if the particle lies out of the object of the camera we can see an interferogram image. The example of such obtained image of captured particles by this method is shown in the figure 11. The diameter of the interferogram corresponds to the particle towards the object plane, the number of lanes in the interferogram, or their spatial frequency corresponds to the particle size.

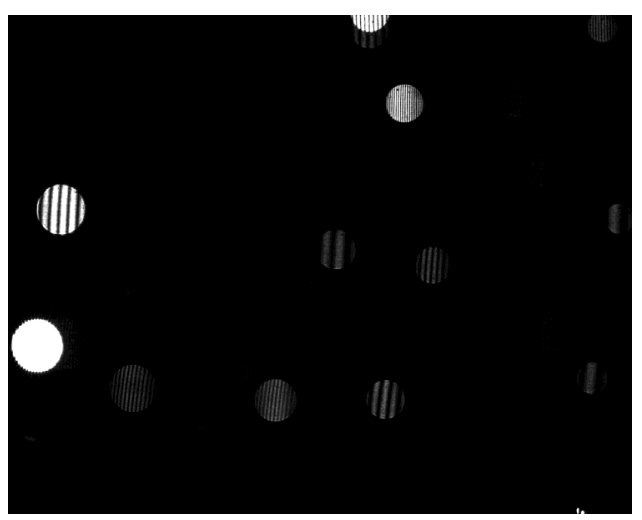

Figure 11. Interferogram image of photographed particles.

Due to the size of the experimental tunnel which was not originally designed for this particular case properties are not the data from IPI method for small particles very accurate, but for bigger diameters it has been observed that the diameter of escaping particles was not higher than approximately 80 microns for the velocity regime of $2.6 \mathrm{~m} / \mathrm{s}$. This result corresponds sufficiently with the numbers obtained from the CFD simulations.

\section{Conclusions}

A profound comparison between numerical and experimental results of the mixed flow in the cooling tower drift eliminator has been done in order to validate the CFD methods for this application. Modern CFD is a very fast and effective tool that can significantly help with design or optimization in this field and the reliability of this approach has been proved in terms of flow field, pressure loss and finally with the efficiency as well. Considering problems with exact geometry fabrication and experimental setup limitations, the results of this comparison are pretty reasonable.

\section{References}

1. J. Čížek, L. Nováková, J. Nožička, Reducing Liquid Phase Drift from Cooling Towers, (Gradient, Prague 2009)

2. T. Hyhlík, EPJ Web of Conferences, 92, (2015), 02027

3. P. Vitkovič, V. Syrovátka, Journal of Flow Visualization and Image Processing, 16(4), (2009), pp. $367-375$

4. J. Stodůlka, R. Vitkovičová, EPJ Web of Conferences, 92, (2015), 02086

5. R. Vitkovičová, P. Vitkovič, J. Čížek, Modifikace metody IPI pro určování velikosti úletu z chladicich věží, SKMTaT, Ústí nad Labem, 2015

\section{Acknowledgement}

This work has been supported by Technology Agency of the Czech Republic under the project Advanced Technologies for Heat and Electricity Production TE01020036 